\title{
Histological correlates of hippocampal magnetization transfer images in drug-resistant temporal lobe epilepsy patients
}

\author{
Jose Eduardo Peixoto-Santos ${ }^{\text {a,b, }}{ }^{*}, 1$, Tonicarlo R. Velasco ${ }^{\mathrm{b}}$, Carlos Gilberto Carlotti ${ }^{\mathrm{c}}$, \\ Joao Alberto Assirati ${ }^{\mathrm{c}}$, Gustavo Henrique de Souza e Rezende ${ }^{\mathrm{d}}$, Katja Kobow ${ }^{\mathrm{e}}$, Roland Coras ${ }^{\mathrm{e}}$, \\ Ingmar Blümcke ${ }^{\mathrm{e}}$, Carlos Ernesto Garrido Salmon ${ }^{\mathrm{f}}$, Antonio Carlos dos Santos ${ }^{\mathrm{g}}$, \\ Joao Pereira Leite ${ }^{b, 2}$

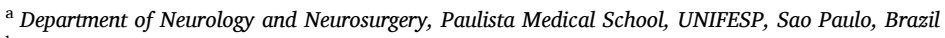 \\ ${ }^{\mathrm{b}}$ Department of Neurosciences and Behavioral Sciences, Ribeirao Preto Medical School, University of Sao Paulo, Ribeirao Preto, Brazil \\ ${ }^{\mathrm{c}}$ Department of Surgery and Anatomy, Ribeirao Preto Medical School, University of Sao Paulo, Ribeirao Preto, Brazil \\ ${ }^{\mathrm{d}}$ Center for Technology and Research in Magneto-Resonance (CTPMAG), Department of Physiology and Biophysics, Federal University of Minas Gerais, Belo Horizonte, \\ Brazil \\ ${ }^{\mathrm{e}}$ Department of Neuropathology, University Hospital Erlangen, Erlangen, Germany \\ ${ }^{\mathrm{f}}$ Department of Physics and Mathematics, Faculty of Philosophy, Science and Languages of Ribeirao Preto, University of Sao Paulo, Ribeirao Preto, Brazil \\ ${ }^{\mathrm{g}}$ Department of Internal Medicine, Ribeirao Preto Medical School, University of Sao Paulo, Ribeirao Preto, Brazil
}

\section{A R T I C L E I N F O}

\section{Keywords:}

Temporal lobe epilepsy

Hippocampal sclerosis

Magnetization transfer ratio

Extracellular matrix

Neuron density

\begin{abstract}
A B S T R A C T
Objective: Temporal lobe epilepsy patients (TLE) often present with hippocampal atrophy, increased T2 relaxation, and reduced magnetization transfer ratio (MTR) in magnetic resonance images (MRI). The histological correlates of the reduced hippocampal MTR are so far unknown. Since MTR is dependent on the tissue's macromolecules, our aim was to evaluate the correlations between cellular populations, extracellular matrix molecules and the MTR in TLE patients.

Methods: Patients with TLE $(n=26)$ and voluntaries $(=20)$ were scanned in a 3 Tesla MRI scanner, and MTR images were calculated from 3DT1 sequences with magnetization pulse on resonance. Immunohistochemistry for neurons, reactive astrocytes, activated microglia, and extracellular matrix chondroitin sulfate were performed in formalin fixed, paraffin embedded tissues of TLE and autopsy controls $(n=10)$. Results were considered significant with adjusted $\mathrm{p}<0.05$.

Results: Compared to the respective controls, TLE patients had reduced hippocampal MTR, increased reactive astrocytes and activated microglia, increased extracellular chondroitin sulfate, and reduced neuron density, compares to controls. MTR correlated positively with neuron density in CA3 and with chondroitin sulfate in CA3 and CA1. Multiple linear regressions reinforced the correlations between chondroitin sulfate and MTR.

Significance: Our data indicate that extracellular matrix molecules are the most significant histological correlates of magnetization transfer ratio in the hippocampus of TLE patients.
\end{abstract}

Abbreviations: ANOVA, Analysis of variance; CA, Cornu Ammonis; CSPG, Chondroitin sulfate proteoglycan; GFAP, Glial fibrillary acid protein; HLA-DR, Human

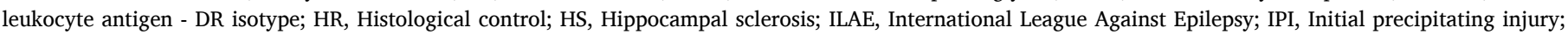

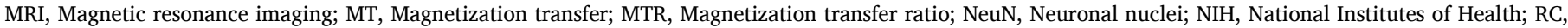
Radiological control; TLE, Temporal lobe epilepsy; VIF, Variance inflation factor.

* Corresponding author at: Department of Neurology and Neurosurgery, Paulista Medical School, UNIFESP, Sao Paulo, Brazil..

E-mail addresses: peixoto.santos@unifesp.br (J.E. Peixoto-Santos), tvelasco@fmrp.usp.br (T.R. Velasco), carlotti@fmrp.usp.br (C.G. Carlotti), assiratijamj@uol,

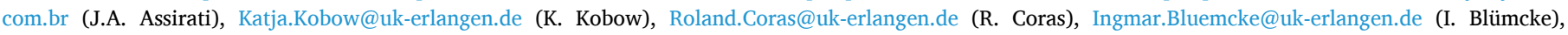
garrido@ffclrp.usp.br (C.E.G. Salmon), acsantos@fmrp.usp.br (A.C. Santos), jpleite@fmrp.usp.br (J.P. Leite).

1 ORCID: 0000-0001-7461-1902.

2 ORCID: 0000-0003-0558-3519. 
Table 1

Quantitative and qualitative MRI evaluation on all cases.

\begin{tabular}{|c|c|c|c|c|c|c|c|c|c|c|c|c|}
\hline \multirow[t]{3}{*}{ Case } & \multirow[t]{3}{*}{ Visual Analysis of the hippocampus } & \multicolumn{9}{|c|}{ Quantitative evaluation of the hippocampus } & \multirow{3}{*}{$\begin{array}{l}\text { HS type in } \\
\text { histology }\end{array}$} & \multirow[t]{3}{*}{ Outcome } \\
\hline & & \multicolumn{3}{|c|}{ Ipsilateral } & \multicolumn{3}{|c|}{ Contralateral } & \multicolumn{3}{|c|}{ Asymmetry } & & \\
\hline & & Volume & $\begin{array}{l}\mathrm{T} 2 \\
\text { relaxation }\end{array}$ & MTR & Volume & $\begin{array}{l}\mathrm{T} 2 \\
\text { relaxation }\end{array}$ & MTR & Volume & Relaxation & MTR & & \\
\hline 1 & $\begin{array}{l}\text { Asymmetry, with ipsilateral } \\
\text { volume reduction and long TR } \\
\text { hypersignal }\end{array}$ & 2.30 & 138.79 & 48.31 & 3.00 & 115.03 & 52.82 & 0.77 & 1.21 & 0.91 & HS1 & ILAE1 \\
\hline 2 & $\begin{array}{l}\text { Asymmetry, with ipsilateral } \\
\text { volume reduction and long TR } \\
\text { hypersignal }\end{array}$ & 3.14 & 86.80 & 48.07 & 3.82 & 80.73 & 49.14 & 0.82 & 1.08 & 0.98 & HS1 & ILAE1 \\
\hline 3 & $\begin{array}{l}\text { Asymmetry, with ipsilateral } \\
\text { volume reduction and long TR } \\
\text { hypersignal }\end{array}$ & 2.10 & 97.58 & 44.54 & 3.10 & 88.60 & 45.77 & 0.68 & 1.10 & 0.97 & HS1 & ILAE2 \\
\hline 4 & $\begin{array}{l}\text { Asymmetry, with ipsilateral } \\
\text { volume reduction and long TR } \\
\text { hypersignal }\end{array}$ & 2.50 & 108.21 & 50.22 & 4.00 & 100.20 & 50.87 & 0.63 & 1.08 & 0.99 & HS1 & ILAE2 \\
\hline 5 & $\begin{array}{l}\text { Asymmetry, with ipsilateral } \\
\text { volume reduction and long TR } \\
\text { hypersignal }\end{array}$ & 1.50 & 131.97 & 40.69 & 3.20 & 116.49 & 41.34 & 0.47 & 1.13 & 0.98 & HS1 & ILAE1 \\
\hline 6 & $\begin{array}{l}\text { Asymmetry, with ipsilateral } \\
\text { volume reduction and long TR } \\
\text { hypersignal }\end{array}$ & 2.84 & 119.80 & 48.46 & 3.94 & 114.32 & 50.08 & 0.72 & 1.05 & 0.97 & HS1 & ILAE1 \\
\hline 7 & $\begin{array}{l}\text { Asymmetry, with long TR } \\
\text { hypersignal and loss of internal } \\
\text { structure }\end{array}$ & 3.58 & 124.00 & 40.92 & 3.87 & 94.75 & 37.62 & 0.92 & 1.31 & 1.09 & HS1 & ILAE3 \\
\hline 8 & $\begin{array}{l}\text { Asymmetry, with ipsilateral } \\
\text { volume reduction, loss of internal } \\
\text { structure, and discrete long TR } \\
\text { hypersignal }\end{array}$ & 1.94 & 154.27 & 42.30 & 3.98 & 127.87 & 46.36 & 0.49 & 1.21 & 0.91 & HS1 & ILAE3 \\
\hline 9 & $\begin{array}{l}\text { Asymmetry, with ipsilateral } \\
\text { volume reduction and long TR } \\
\text { hypersignal }\end{array}$ & 3.63 & 106.03 & 49.68 & 4.41 & 98.78 & 50.79 & 0.82 & 1.07 & 0.98 & HS1 & ILAE1 \\
\hline 10 & $\begin{array}{l}\text { Asymmetry, with ipsilateral } \\
\text { volume reduction and discrete long } \\
\text { TR hypersignal }\end{array}$ & 2.62 & 131.71 & 47.89 & 3.66 & 109.17 & 49.92 & 0.72 & 1.21 & 0.96 & HS1 & ILAE4 \\
\hline 11 & $\begin{array}{l}\text { Asymmetry, with ipsilateral } \\
\text { volume reduction and long TR } \\
\text { hypersignal }\end{array}$ & 2.83 & 111.51 & 49.43 & 4.19 & 109.11 & 50.15 & 0.68 & 1.02 & 0.99 & HS1 & ILAE3 \\
\hline 12 & $\begin{array}{l}\text { Asymmetry, with discrete } \\
\text { ipsilateral volume reduction and } \\
\text { discrete long TR hypersignal }\end{array}$ & 3.70 & 105.88 & 50.88 & 4.90 & 102.34 & 50.21 & 0.76 & 1.03 & 1.01 & HS1 & ILAE1 \\
\hline 13 & $\begin{array}{l}\text { Asymmetry, with ipsilateral } \\
\text { volume reduction and discrete long } \\
\text { TR hypersignal }\end{array}$ & 2.06 & 150.22 & 46.73 & 3.72 & 136.58 & 47.78 & 0.55 & 1.10 & 0.98 & HS1 & ILAE2 \\
\hline 14 & $\begin{array}{l}\text { Asymmetry, with ipsilateral } \\
\text { volume reduction and long TR } \\
\text { hypersignal }\end{array}$ & 2.43 & 129.64 & 47.46 & 3.94 & 106.82 & 50.59 & 0.62 & 1.21 & 0.94 & HS1 & ILAE3 \\
\hline 15 & $\begin{array}{l}\text { Asymmetry, with ipsilateral } \\
\text { volume reduction and discrete long } \\
\text { TR hypersignal }\end{array}$ & 3.08 & 107.60 & 50.47 & 3.90 & 105.89 & 49.88 & 0.79 & 1.02 & 1.01 & HS1 & ILAE1 \\
\hline 16 & $\begin{array}{l}\text { Asymmetry, with ipsilateral } \\
\text { volume reduction and long TR } \\
\text { hypersignal }\end{array}$ & 2.53 & 134.03 & 46.91 & 4.75 & 108.19 & 49.98 & 0.53 & 1.24 & 0.94 & HS1 & ILAE1 \\
\hline 17 & $\begin{array}{l}\text { Asymmetry, with discrete } \\
\text { ipsilateral volume reduction and } \\
\text { normal long TR signal }\end{array}$ & 3.59 & 110.61 & 49.02 & 4.29 & 105.99 & 50.10 & 0.84 & 1.04 & 0.98 & HS1 & ILAE1 \\
\hline 18 & $\begin{array}{l}\text { Asymmetry, with ipsilateral } \\
\text { volume reduction and long TR } \\
\text { hypersignal }\end{array}$ & 3.99 & 113.50 & 49.43 & 4.78 & 108.54 & 50.72 & 0.83 & 1.05 & 0.97 & HS1 & ILAE3 \\
\hline 19 & $\begin{array}{l}\text { Asymmetry, with discrete } \\
\text { ipsilateral volume reduction and } \\
\text { discrete long TR hypersignal in the } \\
\text { hippocampal head }\end{array}$ & 5.01 & 129.41 & 49.44 & 5.51 & 107.36 & 51.40 & 0.91 & 1.21 & 0.96 & HS2 & ILAE3 \\
\hline 20 & $\begin{array}{l}\text { Asymmetry, with ipsilateral } \\
\text { volume reduction and long TR } \\
\text { hypersignal }\end{array}$ & 3.69 & 98.11 & 50.74 & 4.08 & 95.52 & 50.71 & 0.90 & 1.03 & 1.00 & HS2 & ILAE3 \\
\hline 21 & $\begin{array}{l}\text { Asymmetry, with ipsilateral } \\
\text { volume reduction and long TR } \\
\text { hypersignal }\end{array}$ & 1.86 & 137.01 & 44.08 & 3.69 & 116.25 & 47.85 & 0.50 & 1.18 & 0.92 & HS1 & ILAE1 \\
\hline 22 & $\begin{array}{l}\text { Asymmetry, with ipsilateral } \\
\text { volume reduction and long TR } \\
\text { hypersignal }\end{array}$ & 2.40 & 132.81 & 49.41 & 3.20 & 121.34 & 49.81 & 0.75 & 1.09 & 0.99 & HS1 & ILAE1 \\
\hline 23 & $\begin{array}{l}\text { Asymmetry, with discrete } \\
\text { ipsilateral volume reduction, }\end{array}$ & 3.83 & 124.26 & 48.81 & 5.64 & 111.27 & 50.47 & 0.68 & 1.12 & 0.97 & HS1 & ILAE1 \\
\hline
\end{tabular}


Table 1 (continued)

\begin{tabular}{|c|c|c|c|c|c|c|c|c|c|c|c|c|}
\hline \multirow[t]{3}{*}{ Case } & \multirow[t]{3}{*}{ Visual Analysis of the hippocampus } & \multicolumn{9}{|c|}{ Quantitative evaluation of the hippocampus } & \multirow{3}{*}{$\begin{array}{l}\text { HS type in } \\
\text { histology }\end{array}$} & \multirow[t]{3}{*}{ Outcome } \\
\hline & & \multicolumn{3}{|c|}{ Ipsilateral } & \multicolumn{3}{|c|}{ Contralateral } & \multicolumn{3}{|c|}{ Asymmetry } & & \\
\hline & & Volume & $\begin{array}{l}\mathrm{T} 2 \\
\text { relaxation }\end{array}$ & MTR & Volume & $\begin{array}{l}\mathrm{T} 2 \\
\text { relaxation }\end{array}$ & MTR & Volume & Relaxation & MTR & & \\
\hline & $\begin{array}{l}\text { discrete long TR hypersignal, and } \\
\text { loss of internal structure }\end{array}$ & & & & & & & & & & & \\
\hline 24 & $\begin{array}{l}\text { Asymmetry, with ipsilateral } \\
\text { volume reduction and long TR } \\
\text { hypersignal }\end{array}$ & 3.17 & 112.66 & 51.61 & 4.26 & 104.51 & 51.05 & 0.74 & 1.08 & 1.01 & HS1 & ILAE1 \\
\hline 25 & $\begin{array}{l}\text { Asymmetry, with ipsilateral } \\
\text { volume reduction and long TR } \\
\text { hypersignal }\end{array}$ & 2.98 & 141.83 & 47.79 & 4.10 & 111.20 & 49.65 & 0.73 & 1.28 & 0.96 & HS1 & ILAE2 \\
\hline 26 & $\begin{array}{l}\text { Asymmetry, with ipsilateral } \\
\text { volume reduction and long TR } \\
\text { hypersignal }\end{array}$ & 3.10 & 119.09 & 47.93 & 3.80 & 80.70 & 54.75 & 0.82 & 1.48 & 0.88 & HS2 & ILAE1 \\
\hline
\end{tabular}

\section{Introduction}

Hippocampal sclerosis (HS) is the most common pathological finding in adults with drug-resistant epilepsy (Blumcke et al., 2017). In the pathological evaluation, HS is characterized by differential neuron loss, often more severe in CA1 and CA4 (Blumcke et al., 2013). Besides neuron loss, gliosis and changes in extracellular matrix (ECM) proteins are seen in the sclerotic hippocampus of these patients (Crespel et al., 2002; Van Paesschen et al., 1997; Perosa et al., 2002; Peixoto-Santos et al., 2015). Moreover, gliosis can even be seen in the hippocampi of temporal lobe epilepsy patients (TLE) without neuron loss (Thom, 2014). The presurgical magnetic resonance imaging (MRI) of these patients often shows hippocampal volume loss and increased T2 signal (Berkovic et al., 1991; Briellmann et al., 2002; Cendes et al., 1993). However, in up to $15 \%$ of the TLE patients, no abnormalities are observed in the standard MR evaluation (Bernasconi et al., 2000; Jackson et al., 1994). Although the normal MRI patients often have only milder or no neuron loss, some can have neuron loss and other pathological changes as severe as those cases with hippocampal sclerosis detected on MRI (Peixoto-Santos et al., 2015; Jackson et al., 1994). Since the presence of HS is often an indicator of good surgical outcome in TLE (Blumcke et al., 2013; Thom, 2014), it is crucial to improve the presurgical detection of those MRI-negative cases with pathologically proven HS, as well as more subtle pathological changes in the hippocampus.

New quantitative MRI techniques could improve the detection of more subtle abnormalities seen in epilepsy patients (Diniz et al., 2011), especially in those cases were quantitative measurements in the more traditional T1- and T2-weighted images are subtle or absent. Magnetic transfer ratio (MTR), an imaging protocol that evaluates macromolecules not visible in either T1-weighted or T2-weighted images, is often reduced in regions with EEG abnormalities (Rugg-Gunn et al., 2003). While some studies have indicated the association between myelin loss and MTR reduction in the white matter, and between neuron loss and MTR reduction in the cerebral cortex, there remains to be determined which pathological changes are responsible for the decreased hippocampal MTR in the hippocampus of TLE patients. We previously found that ECM chondroitin sulfate proteoglycan (CSPG) could impact both hippocampal volume (Peixoto-Santos et al., 2015) and T2 signal relaxation time (Peixoto-Santos et al., 2017). Since MTR is influenced by the macromolecules present in the tissue, our objective was to evaluate the correlations between the MTR and chondroitin sulfate, as well as with cellular populations, in the hippocampus of drug-resistant temporal lobe epilepsy patients.

\section{Materials and Methods}

\subsection{Patients}

Twenty-six drug-resistant TLE patients were selected during the presurgical evaluation at the Epilepsy Surgery Centre (CIREP) of Ribeirao Preto Medical School. Presurgical workup included history review, neurological evaluation, neuropsychiatric memory tests, videoEEG, and an optimized MRI protocol for TLE, for the definition of epileptogenic focus. Patients with undoubted seizure focus underwent standard en bloc temporal lobe resection.

Thirty age-matched control cases consisted on: twenty healthy volunteers that underwent the same presurgical MRI protocol used for TLE cases (radiological controls, RC), used for defining MRI differences in TLE; ten autopsy cases (histological controls, HC) whose hippocampi were collected to serve as a standard for immunohistochemistry analysis.

Inclusion criteria were: age between 20 and 60 years for all groups; diagnosis of drug-resistant TLE (for TLE group); quantitative MRI protocol (for TLE and RC). Exclusion criteria were: the presence of MRI abnormalities (for RC); generalized or extratemporal EEG spikes for TLE (Cendes et al., 1996, 2000); the presence of pathological changes in the hippocampus in histological evaluation (for HC); the presence of other brain pathology than hippocampal sclerosis (for TLE cases); postmortem time superior to $12 \mathrm{~h}$ (for HC; this cutoff is based on previous study showing stable protein expression up to $12 \mathrm{~h}$ (Peixoto-Santos et al., 2012).

This study was registered in the Brazilian's Health Ministry and approved by the local Research Ethics Committee of the Hospital das Clinicas (HCRP \# 7200/2016). A written Informed Consent Term, previously approved by the Research Ethics Committee, was obtained from all patients or next-of-kin enrolled in this study, following the Declaration of Helsinki.

\subsection{MRI protocol}

All TLE cases and RC volunteers underwent MRI in a Philips Achieva 3.0 T X-series with an 8 elements phase-array head coil. For the definition of hippocampal atrophy, 3D single shot T1-weighted images were performed $\left(\mathrm{TE}=3.2 \mathrm{~ms}\right.$; $\mathrm{TR}=7 \mathrm{~ms}$; flip angle $=8^{\circ}$; inversion pulse $=$ $900 \mathrm{~ms}$; shot interval $=2500 \mathrm{~ms}$; voxel size $=1 \mathrm{~mm}^{3} ; \mathrm{FOV}=240 \times 240$ $\mathrm{mm}$; acquisition time $=4.5 \mathrm{~min})$. $\mathrm{T} 2$ relaxation was additionally measured with 2D turbo spin echo sequences (TEs $=20,40,60,80,100$ $\mathrm{ms}$; TR $=3000 \mathrm{~ms}$; flip angle $=90^{\circ}$; EPI factor $=5$; voxel size $=1 \times 1 \times 3$ $\mathrm{mm}$; FOV $=240 \times 180 \mathrm{~mm}$; acquisition time $=4 \mathrm{~min}$ ). Magnetization transfer ratio was evaluated with two 3D sequences with minimal $\mathrm{T} 1$ and T2 weighting (TFE $=3$; $\mathrm{TE}=3 \mathrm{~ms}$; $\mathrm{TR}=3.6 \mathrm{~ms}$; flip angle $=8^{\circ}$; magnetization transfer saturation pulse on resonance; voxel $=1 \mathrm{x} 1 \mathrm{x} 3$ $\mathrm{mm} ; \mathrm{FOV}=240 \times 180$, acquisition time $=5 \mathrm{~min}$ ). 
The hippocampus was previously segmented using FreeSurfer package (v. 5.3) and 3D-T1w images. The default command recon-all was used for the automatic segmentation of subcortical structures. The segmented hippocampal label was visually double-checked to avoid CSF contamination. The corrected label was used for calculating whole hippocampal volume in the T1 images and was also overlap in the coregistered MTR and T2 maps (calculated with homemade scripts) for extraction of whole hippocampal MTR and T2 values. Absolute hippocampal volume was considered low if below $2.5 \mathrm{~cm}^{3}$, similar to previous studies of our group (Peixoto-Santos et al., 2015), while T2 relaxation was considered abnormal if above average $\left(\mathrm{x}^{-}\right)+2$ standard deviations (s) of controls (i.e., $115.8 \mathrm{~ms}$; controls $=104.7 \pm 5.5 \mathrm{~ms}$ ) and MTR was abnormal if below $\mathrm{x}^{-}-2 \mathrm{~s}$ of controls (i.e., $48.6 \%$; controls $=50.4 \pm$ $0.9 \%)$.

To compare the quantitative results with the visual diagnosis made by expert radiologists (Table 1), we calculated the asymmetry between the ipsilateral and contralateral hippocampus (ipsilateral/contralateral) and compared it with control values. Control asymmetry was defined by randomly assigning either left or right hippocampus as "ipsilateral" and "contralateral", calculating the ipsilateral/contralateral ratio and defining the cutoff as $\mathrm{x}^{-}-2 \mathrm{~s}$ for volume and MTR or $\mathrm{x}^{-}+2 \mathrm{~s}$ for relaxation time. Thus, cutoffs were 0.85 for volume, 0.98 for MTR, and 1.09 for relaxation time. We also compared asymmetry based on a fixed $5 \%$ difference between ipsilateral and contralateral.

\subsection{Immunohistochemistry protocol}

Coronal sections from the hippocampal body of TLE and HC cases were fixed in formalin, dehydrated, clarified, and embedded in paraffin. Eight-micrometer-thick sections were submitted to immunohistochemistry with anti-NeuN (\#MAB377, Chemicon; a neuronal marker), antiGFAP (\#M0761, Dako; a marker of reactive astrocytes), anti-HLA-DR (\#M0746, Dako; a marker of activated microglia), and anti-CSPG (\#C8035, Sigma; a broad-spectrum chondroitin sulfate proteoglycan marker), following protocols and dilutions previously published (Peixoto-Santos et al., 2017).

Micrographs from the regions of interest were collected with an AxioCamMR5 in an Axio Imager M1 microscope with the AxioVision 4.8.1 software (Zeiss). Illumination was maintained constant ( $3 \mathrm{~V}$ ), and exposure varied from each protein ( $33 \mathrm{~ms}$ for NeuN and GFAP, $60 \mathrm{~ms}$ for HLA-DR, and $40 \mathrm{~ms}$ for CSPG), and micrographs were taken at $100 \mathrm{x}$ magnification for NeuN, GFAP, and CSPG, and at 200x magnification for HLA-DR. The analysis was performed with ImageJ $1.45 \mathrm{~s}$ software (NIH), with a semi-quantitative analysis of the immunopositive area fraction (Peixoto-Santos et al., 2017; Eriksson et al., 2007; Kandratavicius et al., 2015). The thresholds used were $154 \pm 10$ for GFAP, $50 \pm 10$ for HLA-DR, and 132 for CSPG. Neuron density was estimated following Abercrombie's method, as described elsewhere (PeixotoSantos et al., 2018). The TLE cases were classified according to the hippocampal sclerosis type (Blumcke et al., 2013). The regions of interest where the hippocampal subfields CA4, CA3, CA2, CA1, and subiculum, as delineated by ILAE Taskforce (Blumcke et al., 2013).

\subsection{Statistics}

For the parametric variables, ANOVA with Bonferroni post hoc test or Student's $t$-test were performed, whereas Kruskal-Wallis with Dunn post hoc test or Mann-Whitney's test were used for non-parametric data. Spearman's correlation was performed to evaluate the association between histological and MR data, and multiple linear models were constructed based on the most relevant results. The association between categorical data and MTR class was explored with logistic regression followed by a $\chi^{2}$ test. All p-values were corrected for false discovery ratio (FDR), and differences were considered significant at adjusted $\mathrm{p}<0.05$.
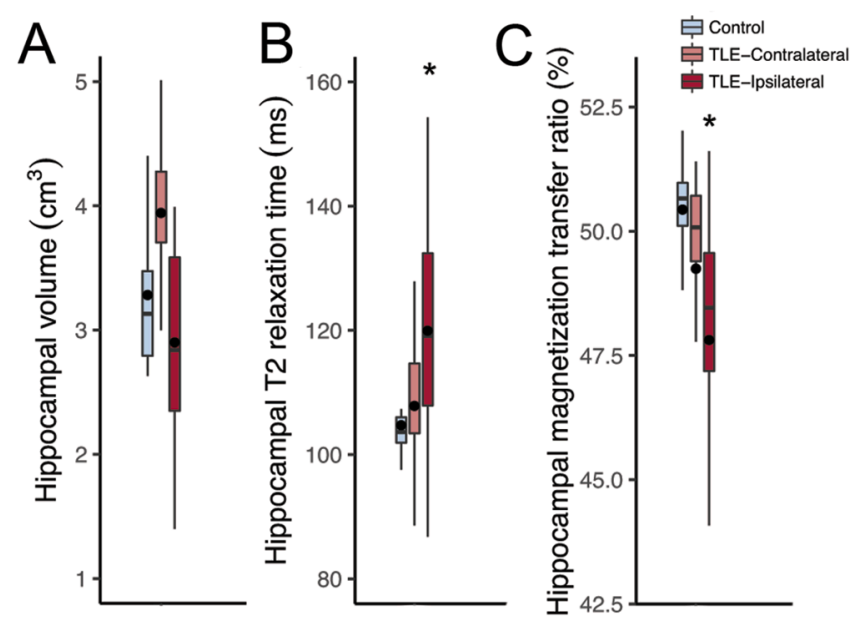

Fig. 1. Quantitative magnetic resonance evaluation of the hippocampi from controls (radiological controls, blue boxplots) and TLE patients (red boxplots). (A) There was no difference between controls and ipsilateral (dark red boxplot) or contralateral (light red boxplot) hippocampi from TLE regarding volume. (B) Only the ipsilateral hippocampus of TLE patients presented with increased T2 relaxation time and $(C)$ reduced magnetization transfer, when compared to controls. There was no difference between the ipsilateral and contralateral hippocampus of TLE cases. The asterisks indicate statistical difference, the line inside the boxplots indicate median and the dot indicate mean. (For interpretation of the references to colour in this figure legend, the reader is referred to the web version of this article.) (For interpretation of the references to colour in this figure legend, the reader is referred to the web version of this article.)

\section{Results}

\subsection{Clinical data}

All subject enrolled in the present study were age-matched $(\mathrm{HC}=$ $46.9 \pm 13.6$ years, $\mathrm{RC}=43.6 \pm 7.6$ years, and $\mathrm{TLE}=43.2 \pm 11.8$ years, ANOVA). Postmortem interval was of $8.3 \pm 4.1 \mathrm{~h}$ (ranging from 3 to 12 h), and most HC cases had heart failure as the main cause of death (50\%), followed by sepsis (30\%) and pneumonia (20\%). TLE cases had the first seizure at $10.1 \pm 10.0$ years (median of 5.5 years, ranging from 0.5 to 35 years) and seizure recurrence at $18.0 \pm 11.1$ years (median of 17 years, ranging from 0.5 to 45 years). All remaining clinical data are described in Table 1.

\subsection{MRI evaluation}

The automatic hippocampal analysis showed no significant difference in hippocampal volume between controls and TLE cases (Studens, $\mathrm{p}=0.169$; Fig. 1 A). Only nine of the TLE patients (34.6\%) had hippocampal atrophy, following the cutoffs of previous quantitative studies with a different set of cases (Peixoto-Santos et al., 2015). Hippocampus from TLE patients presented with higher relaxation time than controls (Mann-Whitney, p < 0.001; Fig. 1 B). The hippocampi from TLE cases had a significant reduction in MTR compared to controls (Mann-Whitney, $\mathrm{p}<0.001$; Fig. 1 C). Based on control hippocampi average and standard deviation, cases below 95\% MTR of controls (i.e., below $48.6 \%$ magnetization transfer) were classified as having low MTR, and those above as having normal MTR. In the patients without hippocampal volume loss, the low MTR was seen in 50\% of cases. As for the cases with hippocampal atrophy, $88 \%$ had low MTR. The combination with T2 relaxation increases the detection of the abnormal hippocampus to $64 \%$ of normal volume cases. Finally, from all cases, two presented only MTR reduction, and eight were MRI-negative patients (i.e., normal hippocampal volume, T2 relaxation, and MTR).

Qualitative (visual) evaluation of expert radiologists indicated marked asymmetric hippocampal volume in $84 \%$ of the cases, discrete 


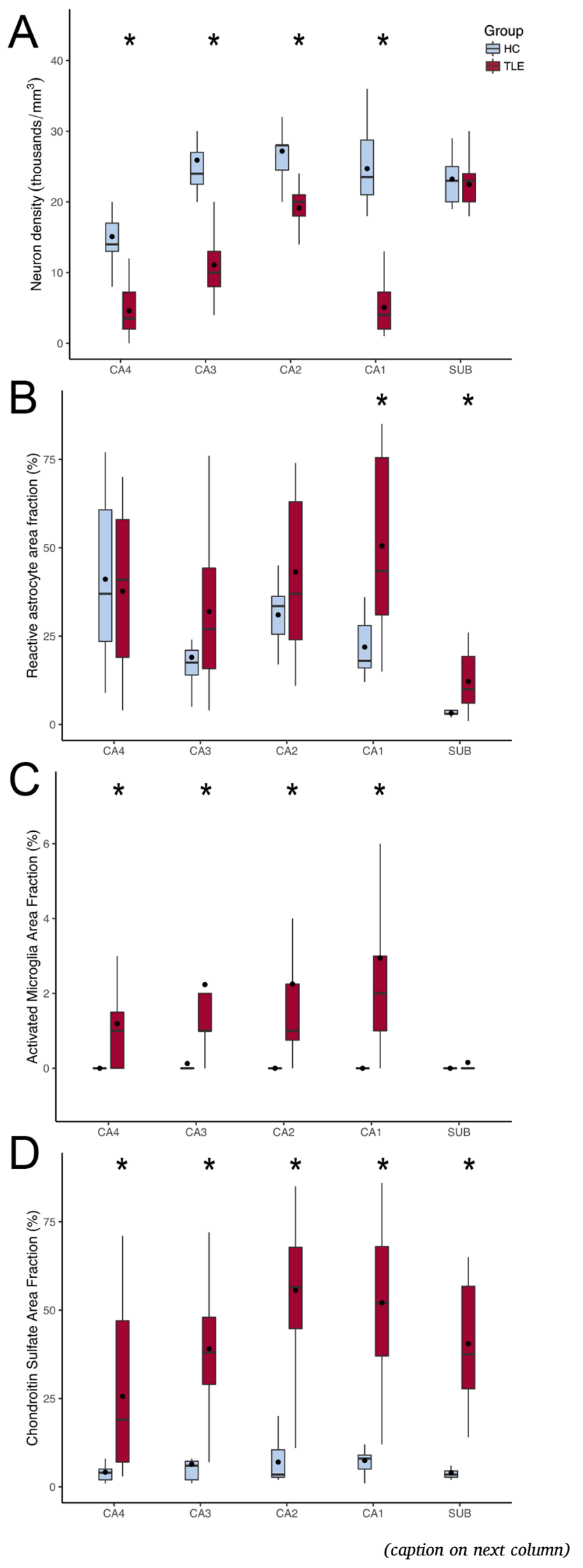

Fig. 2. Semi-quantitative evaluation of hippocampal sections from controls (histological controls, blue boxplots) and TLE patients (red boxplots) submitted to immunohistochemistry. (A) All hippocampal subfields but the subiculum of TLE cases presented with neuron loss. (B) Only CA1 and the subiculum of TLE had significant astroglial reaction, when compared to controls. (C) Following the neuron density changes, all hippocampal subfields but the subiculum had activated microglia, when compared to controls. (D) Chondroitin sulfate proteoglycan was seen in higher levels in all hippocampal subfields of TLE cases, compared to controls. The asterisks indicate statistical difference, the line inside the boxplots indicate median and the dot indicate mean. (For interpretation of the references to colour in this figure legend, the reader is referred to the web version of this article.) (For interpretation of the references to colour in this figure legend, the reader is referred to the web version of this article.)

volume asymmetry in $12 \%$ and normal volume in $4 \%$. Visual T2weighted and FLAIR signal were considered markedly asymmetric in $69 \%$ of the cases, discretely increased in $27 \%$, and normal in $4 \%$. With the combination of qualitative volume and signal evaluation, all subjects had some hippocampal asymmetry (see Table 1). In a similar approach, the quantitative data matched the visual assessment. Based on the controls, the asymmetry indicated that the epileptogenic (ipsilateral) hippocampus was smaller in $88 \%$ of the cases, had increased T2 relaxation in $54 \% \%$ of the patients, and MTR was lower in $50 \%$ of the cases. However, the difference in MTR was more subtle, and a difference higher than $5 \%$ was only seen in $23 \%$ of the cases (versus $100 \%$ with volume and $81 \%$ with T2 relaxation differences higher than $5 \%$ of the contralateral value; Table 1).

\subsection{Cellular populations and extracellular matrix}

TLE patients had lower neuron density than controls in CA4, CA3, CA2, and CA1 (Mann-Witney, $\mathrm{p}<0.001$ ), with no difference in the subiculum (Student, $\mathrm{p}=0.629$; Fig. $2 \mathrm{~A}$ ). Reactive astrogliosis was seen in CA1 (Mann-Whitney, $\mathrm{p}=0.002$ ) and in the subiculum (Mann-Whitney, $\mathrm{p}=0.003$; Fig. 2 B) of TLE cases, compared to controls. Activated microglia was present in CA4 (Mann-Whitney, $\mathrm{p}=0.016$ ), CA3 (MannWhitney, $\mathrm{p}=0.006$ ), CA2 (Mann-Whitney, $\mathrm{p}=0.005$ ), and in CA1 (Mann-Whitney, $\mathrm{p}=0.001$; Fig. 2 C) of TLE patients, compared to controls. Increased expression of chondroitin sulfate proteoglycan was seen in CA4 (Mann-Whitney, $\mathrm{p}=0.002$ ), CA3 (Student, $\mathrm{p}<0.001$ ), CA2 (Mann-Whitney, $\mathrm{p}<0.001$ ), CA1 (Mann-Whitney, $\mathrm{p}<0.001$ ), and in the subiculum (Mann-Whitney, $\mathrm{p}<0.001$; Fig. 2 D). A representative image showing the expression patterns in TLE is shown in Fig. 3.

\subsection{Associations between MRI, clinical data, and histology}

Hippocampal MTR were not significantly different regarding HS type, the presence of focal to bilateral tonic-clonic seizures, the occurrence of status epilepticus or seizures in clusters, a positive familial history of seizures, seizure frequency, occurrence of IPI, or surgical outcome (see Table 2). Additionally, all three HS type 2 had normal hippocampal volume, two had increased T2 relaxation time, and one had lower MTR (Table 1). Logistic regression with the MTR classification (low vs. normal) reinforced the lack of association between MTR and the clinical data.

Hippocampal MTR correlated positively with neuron density in CA3 $(\mathrm{R}=0.581, \mathrm{p}=0.038$; Fig. $4 \mathrm{~A})$ and with CSPG levels in CA3 $(\mathrm{R}=0.66$, $\mathrm{p}=0.038$; Fig. 4 B) and CA1 ( $\mathrm{R}=0.544, \mathrm{p}=0.043$; Fig. 4 C). Negative correlations between hippocampal MTR and activated microglia were seen in CA2 ( $R=-0.636, p=0.043)$, and in CA1 $(R=-0.604, p=$ 0.038). Reactive astrocytes had no correlation with MTR.

From different combinations of neuron density and CSPG levels in CA4, CA3, CA1, and the subiculum, the best multiple linear model for explaining the variation in hippocampal MTR were the combination of neuron density and CSPG in CA3 and CA1, with a overall predictive value of $71 \%$ for the MTR $\left(\mathrm{R}=0.841, \mathrm{R}^{2}=0.708, \mathrm{VIF}<2.971, \mathrm{p}=\right.$ 


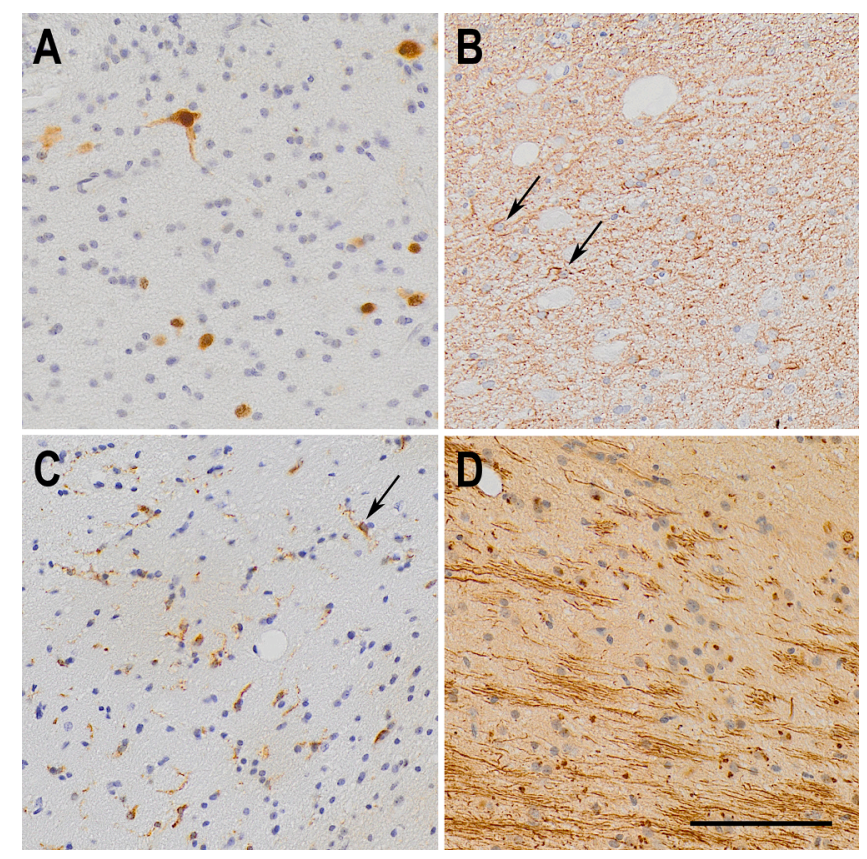

Fig. 3. Representative immunohistochemistry micrographs of the pathological changes seen in the CA1 subfield from TLE samples. (A) NeuN staining, showing reduced neuron density in TLE CA1. (B) GFAP staining showing fibrous astrogliosis, as defined by ILAE, in a TLE case. Two reactive astrocytes are indicated by arrows. (C) Increased activation of microglial cells, marked by the expression of MHC class II HLA-DR protein. One activated microglia is pointed by the black arrow. (D) Fibrous aggregates of extracellular chondroitin sulfate proteoglycans, showed by CS-56 antigen immunohistochemistry. The bar in (D) indicates $100 \mu \mathrm{m}$.

0.006). The following equation was given for these variables:

MTR $=40.282+(0.324 \times$ neuron density in CA3 $)-(0.274 \times$ neuron density in CA1 $)+(0.0750 \times$ CSPG levels in CA3 $)+(0.0429 \times$ CSPG levels in CA1)

Individually, the changes in CA3 explain $59 \%$ of the hippocampal MTR $\left(R=0.767, R^{2}=0.588, V I F=1.049 p=0.003\right)$, whereas CA1 explains $43 \%$ of MTR $\left(R=0.652, R^{2}=0.426\right.$, VIF $\left.=1.035, p=0.009\right)$. The multiple linear regression equations are as follows:

MTR $=39.779+(0.349 \times$ neuron density in CA3 $)+(0.100 \times$ CSPG levels in CA3)

MTR $=43.487-(0.131 \times$ neuron density in CA1 $)+(0.0943 \times$ CSPG levels in CA1)

\section{Discussion}

Reduced hippocampal volume and increased T2-weighted signal are important hallmarks of the epileptogenic hippocampi in drug-resistant TLE patients (Berkovic et al., 1991; Briellmann et al., 2002; Cendes et al., 1993). However, some patients have no MRI abnormalities in the standard MRI sequences (Bernasconi et al., 2000; Jackson et al., 1994). In these cases, new MRI techniques could help improve the MRI detection of the epileptogenic focus. Our present study evaluated MTR in the hippocampus of drug-resistant TLE patients and different hippocampal volumes on MRI, showing reduced MTR in the epileptogenic hippocampi. Half of the patients whose hippocampus had a normal volume on quantitative evaluation presented a reduced MTR, indicating that in normal volume cases MTR could help define the epileptogenic hippocampus. Moreover, in two cases ( $8 \%$ of our TLE) with normal volume and normal T2 relaxation, MTR was below normal levels. However, if an approach closer to the standard visual assessment (i.e., the visual asymmetry between ipsilateral and contralateral) is taken into account to define the epileptogenic hippocampus, MTR was by far the worse
Table 2

MTR value according to clinical data classes.

\begin{tabular}{|c|c|c|c|c|}
\hline Variable & & $\begin{array}{l}\text { Percentage of } \\
\text { TLE cases }\end{array}$ & MTR & $\begin{array}{l}\mathrm{p}- \\
\text { value }\end{array}$ \\
\hline \multirow[t]{2}{*}{ HS type } & HS1 & $88 \%$ & $\begin{array}{l}48.314 \\
(46.730-49.430)^{*}\end{array}$ & 0.696 \\
\hline & HS2 & $12 \%$ & $\begin{array}{l}49.440 \\
(47.927-50.740)\end{array}$ & \\
\hline \multirow{2}{*}{$\begin{array}{l}\text { Focal to bilateral } \\
\text { tonic-clonic } \\
\text { seizures }\end{array}$} & No & $32 \%$ & $\begin{array}{l}48.460 \\
(47.625-50.675)\end{array}$ & 0.684 \\
\hline & Yes & $68 \%$ & $\begin{array}{l}47.890 \\
(42.300-49.415)\end{array}$ & \\
\hline \multirow[t]{2}{*}{ Seizure Clusters } & No & $20 \%$ & $\begin{array}{l}47.350 \\
(42.420-49.800)\end{array}$ & 0.706 \\
\hline & Yes & $80 \%$ & $\begin{array}{l}48.810 \\
(47.460-49.430)\end{array}$ & \\
\hline \multirow[t]{2}{*}{ Status Epilepticus } & No & $76 \%$ & $\begin{array}{l}48.810 \\
(47.460-50.225)\end{array}$ & 0.7 .06 \\
\hline & Yes & $24 \%$ & $\begin{array}{l}47.790 \\
(43.799-50.512)\end{array}$ & \\
\hline \multirow[t]{2}{*}{$\begin{array}{l}\text { Familiar History } \\
\text { of Seizures }\end{array}$} & No & $42 \%$ & $\begin{array}{l}49.020 \\
(47.790-49.680)\end{array}$ & 0.743 \\
\hline & Yes & $58 \%$ & $\begin{array}{l}48.387 \\
(46.182-49.690)\end{array}$ & \\
\hline \multirow[t]{4}{*}{ Seizure Frequency } & Daily & $12 \%$ & $\begin{array}{l}49.430 \\
(48.460-50.880)\end{array}$ & 0.706 \\
\hline & Weekly & $56 \%$ & $\begin{array}{l}47.998 \\
(46.317-49.500)\end{array}$ & \\
\hline & Biweekly & $12 \%$ & $\begin{array}{l}47.460 \\
(46.730-50.470)\end{array}$ & \\
\hline & Monthly & $20 \%$ & $\begin{array}{l}49.020 \\
(44.867-50.512)\end{array}$ & \\
\hline \multirow[t]{2}{*}{ IPI Presence } & No & $54 \%$ & $\begin{array}{l}47.858 \\
(44.425-49.430)\end{array}$ & 0.684 \\
\hline & Yes & $46 \%$ & $\begin{array}{l}49.112 \\
(47.935-50.273)\end{array}$ & \\
\hline \multirow[t]{3}{*}{ Outcome } & ILAE1 & $54 \%$ & $\begin{array}{l}48.635 \\
(47.673-49.877)\end{array}$ & 0.706 \\
\hline & ILAE2 & $14 \%$ & $\begin{array}{l}47.260 \\
(45.087-49.616)\end{array}$ & \\
\hline & ILAE3/4 & $32 \%$ & $\begin{array}{l}48.660 \\
(43.590-49.438)\end{array}$ & \\
\hline
\end{tabular}

* Median (lower quartile - upper quartile).

technique to indicate the epileptogenic hippocampus. Especially if we take into account that the very subtle differences in MTR are likely not detected by visual assessment, this technique is more interesting for research only. Moreover, lower MTR was not associated with any of the clinical variables investigated. Thus, our finding agrees with previous data in which reduced MTR was seen in the hippocampus related to seizure focus (Tofts et al., 1995), but indicates that MTR has very limited use for the lateralization of the focus in TLE cases with hippocampal sclerosis.

The atrophy seen in the epileptogenic hippocampus was linked to the degree of neuron loss in the granule cell layer (Briellmann et al., 2002) but more consistently to neuron loss in the CA1 subfield (Van Paesschen et al., 1997; Diehl et al., 2002). A previous study from our group also pointed out neuron loss in CA1, together with chondroitin sulfate levels in this subfield, as the most important factor for explaining hippocampal volume (Peixoto-Santos et al., 2015). Moreover, this subfield is, together with the subiculum, the largest hippocampal subfield (Peixoto-Santos et al., 2018), and is the most affected both in patients and in animal models (Blumcke et al., 2013; Do Val-da Silva et al., 2016). Reduced magnetization transfer has been linked to both myelin and neuron (axonal) loss in multiple sclerosis (van Waesberghe et al., 1999; Fisher et al., 2007; Mottershead et al., 2003). An animal model of autoimmune encephalomyelitis further indicated that MTR reduction in regions of neuron loss is more evident than in those with only demyelination (Rausch et al., 2009). In TLE, neuron loss is believed to be associated with MTR reduction (Flugel et al., 2006). However, a study with temporal cortex showed no correlation between neuronal population and 

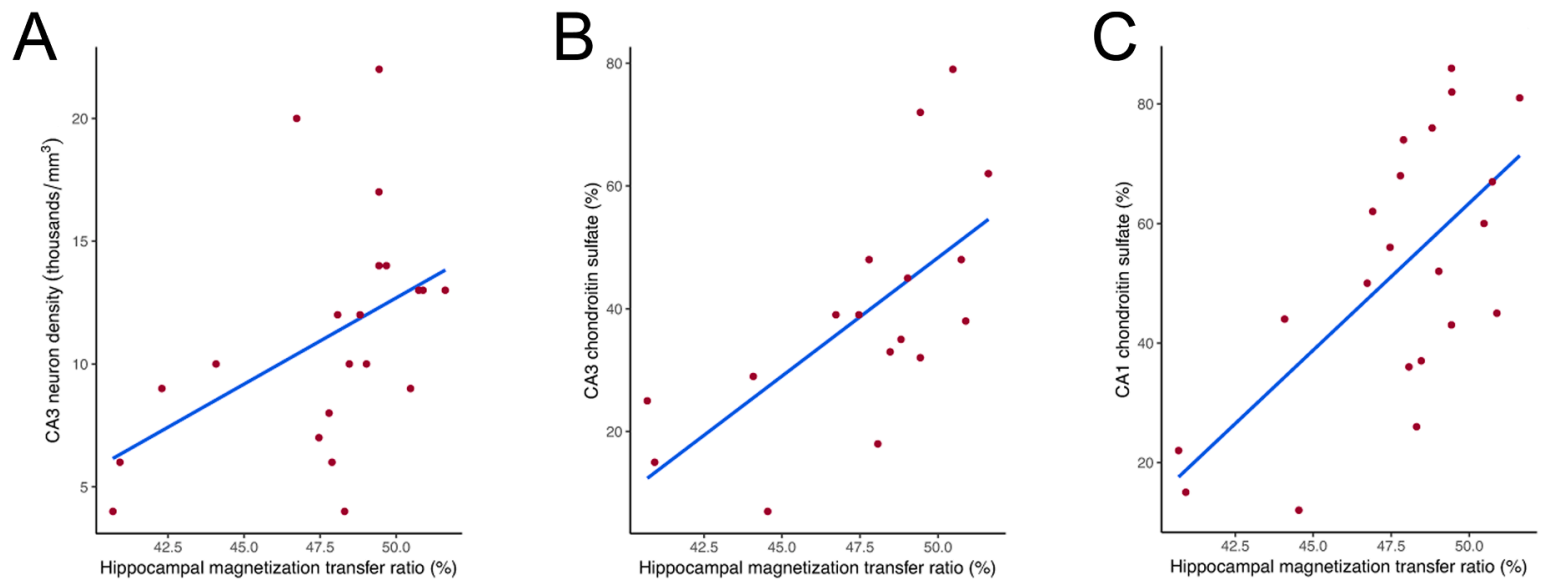

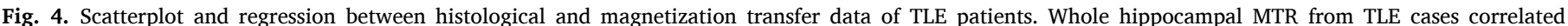
positively with neuron density in CA3 (A) and with chondroitin sulfate levels in CA3 (B), CA1 (C), and the subiculum (D).

MTR values (Eriksson et al., 2007). Our data indicate that neuron loss in CA3 is important for MTR reduction seen in TLE.

From the glial population evaluated with immunohistochemistry, only the activated microglia correlated with the hippocampal MTR. The glial reaction is a common finding in the hippocampus of drug-resistant TLE patients (Peixoto-Santos et al., 2015, 2017; Kandratavicius et al., 2015). Gliosis often following the degree of neuronal loss and seizure severity (Crespel et al., 2002; Khurgel and Ivy, 1996). However, the glial reaction is known to occur in the absence of neuron loss (Thom, 2014). Increased T2 signal and relaxation time is often linked to reactive astrogliosis (Van Paesschen et al., 1997; Briellmann et al., 2002), whereas no MRI sequence so far has been directly linked to microglia (Banati et al., 1999; Doorduin et al., 2009; Kumar et al., 2008). We saw negative correlations between MTR and activated microglia, but not with reactive astrocytes. Given the usually low increase in activated microglia in the hippocampus when compared to the often exuberant neuron loss and astroglial reaction, it would be unexpected for microglia to affect MRI sequences. It is possible that the inverse correlation between microglia and MTR is an indirect effect from the stronger association between neuron loss and CSPG with MTR. In fact, when added to the multiple linear regression model that included CA3 and CA1 values, the activated microglia presents the highest VIF values (2.935 for CA3 and 4.037 for CA1), indicating multicollinearity between microglia and other factors. Thus, we believe activated microglia should be seen as an indirect indicator for MTR changes.

ECM in the central nervous system is often studied concerning tissue plasticity (Silver and Miller, 2004; Huang et al., 2006). Increased CSPG levels were already described in patients with epilepsy and animal models (Perosa et al., 2002; Schwarzacher et al., 2006). In the pathology/MRI correlation field, some studies have shown an association between apparent diffusion coefficient changes and increased levels of CSPG (Roitbak and Sykova, 1999; Vorisek et al., 2002). Given that ECM accounts for $20 \%$ of the brain parenchyma (Davson and Spaziani, 1959; Sykova, 2004), it is expected that these molecules are important for MRI changes. In fact, previous studies of our group with hippocampi of TLE patients have shown a significant association between CSPG and changes in hippocampal volume and T2 relaxation (Peixoto-Santos et al., 2015, 2017). The link between ECM molecules and magnetization transfer is underexplored, and studies have provided mixed results. In phantoms of collagen and CSPG, while a study showed no correlation CSPG and MTR (Seo et al., 1996), another one has shown a weak positive association between CSPG and MTR (Laurent et al., 2001). Studies with animal cartilage also indicated that MTR is more often affected by the amount of collagen, with little effect of CSPG (Laurent et al., 2001; Toffanin et al., 2001). Our present data indicate that hippocampal MTR is strongly associated with the levels of CSPG. Since CSPG are increased in HS and correlate directly with MTR, one should expect an increased, and not decreased MTR in HS. However, neuron density is also directly proportional to MTR and is often lower in TLE. Thus, while neuron loss reduces MTR, increased CSPG increase it, and the balance between both will define if MTR will be within a normal range or significantly decrease. This could explain why some cases with HS type 1, the more severe HS in terms of neuron loss, have average MTR: in such cases, the increased CSPG expression may hide the intensity of neuron loss. A similar effect was seen in a previous study with another subset of patients, where some patients with severe neuron loss presented an average hippocampal volume due to a higher content of CSPG in the ECM (Peixoto-Santos et al., 2015). In summary, the presence of normal hippocampal MTR should not rule out the occurrence of hippocampal neuron loss and, thus, hippocampal sclerosis.

The most important limitation of the present study is, in our view, that we only evaluated the extracellular matrix with a broad-spectrum anti-chondroitin sulfate antibody. Besides the chondroitin sulfate proteoglycans, hyaluronic acid is a major constituent of the ECM (Perosa et al., 2002; Laurent and Fraser, 1992). Given that MTR is affected by macromolecules (Kim et al., 2014), and those ECM are some of the largest molecules in the brain, a more thorough evaluation of other extracellular molecules, such as phosphacan (the molecules that compose the perineuronal nets) and neurocan, as well as hyaluronic acid, should increase the correlation between ECM and MTR. However, ECM molecules are better evaluated with western blot, which was not feasible for our paraffin-embedded samples. An investigation with a more extensive series, especially with more HS type 2 and 3 patients, should evaluate the possible importance of MTR in separating HS types. These HS types are usually not distinguishable in presurgical MRI but can be identified in high-field ex-vivo MRI with smaller voxel size (Peixoto-Santos et al., 2018). Even if more HS type 1 cases where added, we believe stronger associations would emerge. Apart from HS types, we know that even the same HS type can had differential synaptic reorganization in several regions (Dombroski et al., 2020). It would be interesting to see if these, changes could impact MTR in higher field MRI, with smaller voxel sizes and subfield MTR assessment. In our consecutive sections from the hippocampal body immunostained for NeuN, GFAP, HLA-DR, and CSPG, we saw the same type of HS. However, we are aware that some studies indicated a variability of HS type across the hippocampal long axis (Steve et al., 2020; Thom et al., 2012). Although only a few studies have shown this variability, the use of several consecutive slices for the immunohistochemistry evaluation would also be necessary. However, we only had access to slices at the hippocampal body. Another critical problem of the MRI-histology correlational studies that is often not discussed is the problem of associating variables of different dimensionalities (i.e., onemm-thick MRI signal of whole 
hippocampal data with eight- $\mu$ m-thin slices of immunohistochemistry evaluated in ROIs of different subfields). If replaced by wholehippocampus western blot, we would lose subfields variability, but the general picture would be closer to whole hippocampal MTR values. The problem of comparing variables of different dimensions, as far as we know, has no clear, widely accepted solution. A better approach for future studies could be through immunohistochemistry evaluation of the hippocampal subfields on several slices throughout the hippocampus long axis and compare it with discrete MTR values of each subfield, and enrich the data with molecular approaches such as western blot and proteomics. Higher-field MRI with smaller voxels could also improve correlational studies.

\section{Conclusions}

The present study indicates that neuron density and extracellular matrix chondroitin sulfate are associated with MTR changes in the hippocampi of TLE patients, and together can explain more than $70 \%$ of the MTR signal in the ipsilateral hippocampus.

\section{Funding}

This research was funded by Fundacao de Amaparo a Pesquisa do Estado de Sao Paulo (FAPESP), grant numbers 2015/20840-9 and 2016/17882-4.

\section{Acknowledgement}

We would like to thank the excellent technical support of Antonio Renato Meirelles e Silva, Geraldo Cassio dos Reis, and the staff from the CIREP, Magnetic Resonance Unit, Center of Imaging Sciences and Medical Physics (CCIFM), and Pathology Department.

\section{References}

Blumcke, I., Spreafico, R., Haaker, G., Coras, R., Kobow, K., Bien, C.G., et al., 2017. Histopathological Findings in Brain Tissue Obtained during Epilepsy Surgery. The New England J. Med. 377, 1648-1656.

Blumcke, I., Thom, M., Aronica, E., Armstrong, D.D., Bartolomei, F., Bernasconi, A., et al., 2013. International consensus classification of hippocampal sclerosis in temporal lobe epilepsy: a Task Force report from the ILAE Commission on Diagnostic Methods. Epilepsia 54, 1315-1329.

Crespel, A., Coubes, P., Rousset, M.C., Brana, C., Rougier, A., Rondouin, G., et al., 2002. Inflammatory reactions in human medial temporal lobe epilepsy with hippocampal sclerosis. Brain Res. 952, 159-169.

Van Paesschen, W., Revesz, T., Duncan, J.S., King, M.D., Connelly, A., 1997. Quantitative neuropathology and quantitative magnetic resonance imaging of the hippocampus in temporal lobe epilepsy Annals of neurology 42, 756-766.

Perosa, S.R., Porcionatto, M.A., Cukiert, A., Martins, J.R., Passeroti, C.C., Amado, D., et al., 2002. Glycosaminoglycan levels and proteoglycan expression are altered in the hippocampus of patients with mesial temporal lobe epilepsy. Brain Res. Bull. 58, 509-516.

Peixoto-Santos, J.E., Velasco, T.R., Galvis-Alonso, O.Y., Araujo, D., Kandratavicius, L., Assirati, J.A., et al., 2015. Temporal lobe epilepsy patients with severe hippocampal neuron loss but normal hippocampal volume: Extracellular matrix molecules are important for the maintenance of hippocampal volume. Epilepsia 56, 1562-1570.

Berkovic, S.F., Andermann, F., Olivier, A., Ethier, R., Melanson, D., Robitaille, Y., et al., 1991. Hippocampal sclerosis in temporal lobe epilepsy demonstrated by magnetic resonance imaging Annals of neurology 29, 175-182.

Briellmann, R.S., Kalnins, R.M., Berkovic, S.F., Jackson, G.D., 2002. Hippocampal pathology in refractory temporal lobe epilepsy: T2-weighted signal change reflects dentate gliosis. Neurology 58, 265-271. Research Support, Non-U.S. Gov't.

Cendes, F., Andermann, F., Dubeau, F., Gloor, P., Evans, A., Jones-Gotman, M., et al., 1993. Early childhood prolonged febrile convulsions, atrophy and sclerosis of mesial structures, and temporal lobe epilepsy: an MRI volumetric study. Neurology 43, 1083-1087.

Bernasconi, A., Bernasconi, N., Caramanos, Z., Reutens, D.C., Andermann, F., Dubeau, F., et al., 2000. T2 relaxometry can lateralize mesial temporal lobe epilepsy in patients with normal MRI. NeuroImage. 12, 739-746.

Jackson, G.D., Kuzniecky, R.I., Cascino, G.D., 1994. Hippocampal sclerosis without detectable hippocampal atrophy. Neurology 44, 42-46.

Diniz, P.R., Velasco, T.R., Salmon, C.E., Sakamoto, A.C., Leite, J.P., Santos, A.C., 2011 Extratemporal damage in temporal lobe epilepsy: magnetization transfer adds information to volumetric MR imaging. AJNR Am. J. Neuroradiol. 32, 1857-1861.
Rugg-Gunn, F.J., Eriksson, S.H., Boulby, P.A., Symms, M.R., Barker, G.J., Duncan, J.S., 2003. Magnetization transfer imaging in focal epilepsy. Neurology 60, 1638-1645.

Peixoto-Santos, J.E., Kandratavicius, L., Velasco, T.R., Assirati, J.A., Carlotti, C.G., Scandiuzzi, R.C., et al., 2017. Individual hippocampal subfield assessment indicates that matrix macromolecules and gliosis are key elements for the increased T2 relaxation time seen in temporal lobe epilepsy. Epilepsia 58, 149-159.

Cendes, F., Dubeau, F., Andermann, F., Quesney, L.F., Gambardella, A., JonesGotman, M., et al., 1996. Significance of mesial temporal atrophy in relation to intracranial ictal and interictal stereo EEG abnormalities. Brain: A J. Neurol. 119 (Pt 4), 1317-1326.

Cendes, F., Li, L.M., Watson, C., Andermann, F., Dubeau, F., Arnold, D.L., 2000. Is ictal recording mandatory in temporal lobe epilepsy? Not when the interictal electroencephalogram and hippocampal atrophy coincide. Arch. Neurol. 57, 497-500.

Peixoto-Santos, J.E., Galvis-Alonso, O.Y., Velasco, T.R., Kandratavicius, L., Assirati, J.A., Carlotti, C.G., et al., 2012. Increased metallothionein I/II expression in patients with temporal lobe epilepsy. PloS One 7, e44709.

Eriksson, S.H., Free, S.L., Thom, M., Martinian, L., Symms, M.R., Salmenpera, T.M., et al., 2007. Correlation of quantitative MRI and neuropathology in epilepsy surgical resection specimens-T2 correlates with neuronal tissue in gray matter. NeuroImage $37,48-55$.

Kandratavicius, L., Peixoto-Santos, J.E., Monteiro, M.R., Scandiuzzi, R.C., Carlotti Jr., C. G., Assirati Jr, J.A., et al., 2015. Mesial temporal lobe epilepsy with psychiatric comorbidities: a place for differential neuroinflammatory interplay. J. Neuroinflammation $12,38$.

Peixoto-Santos, J.E., de Carvalho, L.E.D., Kandratavicius, L., Diniz, P.R.B., Scandiuzzi, R. C., Coras, R., et al., 2018. Manual Hippocampal Subfield Segmentation Using HighField MRI: Impact of Different Subfields in Hippocampal Volume Loss of Temporal Lobe Epilepsy Patients. Front. Neurol. 9, 927.

Tofts, P.S., Sisodiya, S., Barker, G.J., Webb, S., MacManus, D., Fish, F., et al., 1995. MR magnetization transfer measurements in temporal lobe epilepsy: a preliminary study. AJNR Am. J. Neuroradiol. 16, 1862-1863.

Diehl, B., Najm, I., Mohamed, A., Babb, T., Ying, Z., Bingaman, W., 2002. Interictal EEG, hippocampal atrophy, and cell densities in hippocampal sclerosis and hippocampal sclerosis associated with microscopic cortical dysplasia. Journal of clinical neurophysiology: official publication of the American Electroencephalographic Society. 19, 157-162.

Do Val-da Silva, R.A., Peixoto-Santos, J.E., Scandiuzzi, R.C., 2016. Decreased neuron loss and memory dysfunction in pilocarpine-treated rats pre-exposed to hypoxia. Neuroscience 332, 88-100.

van Waesberghe, J.H., Kamphorst, W., De Groot, C.J., van Walderveen, M.A., Castelijns, J.A., Ravid, R., et al., 1999. Axonal loss in multiple sclerosis lesions: magnetic resonance imaging insights into substrates of disability. Ann. Neurol. 46, $747-754$.

Fisher, E., Chang, A., Fox, R.J., Tkach, J.A., Svarovsky, T., Nakamura, K., et al., 2007. Imaging correlates of axonal swelling in chronic multiple sclerosis brains. Ann. Neurol. 62, 219-228.

Mottershead, J.P., Schmierer, K., Clemence, M., Thornton, J.S., Scaravilli, F., Barker, G. J., et al., 2003. High field MRI correlates of myelin content and axonal density in multiple sclerosis-a post-mortem study of the spinal cord. J. Neurol. 250, 1293-1301.

Rausch, M., Tofts, P., Lervik, P., Walmsley, A., Mir, A., Schubart, A., et al., 2009. Characterization of white matter damage in animal models of multiple sclerosis by magnetization transfer ratio and quantitative mapping of the apparent bound proton fraction f. Mult. Scler. 15, 16-27.

Flugel, D., O’Toole, A., Thompson, P.J., Koepp, M.J., Cercignani, M., Symms, M.R., et al., 2006. A neuropsychological study of patients with temporal lobe epilepsy and chronic interictal psychosis. Epilepsy Res. 71, 117-128.

Khurgel, M., Ivy, G.O., 1996. Astrocytes in kindling: relevance to epileptogenesis. Epilepsy Res. 26, 163-175.

Banati, R.B., Goerres, G.W., Myers, R., Gunn, R.N., Turkheimer, F.E., Kreutzberg, G.W., et al., 1999. [11C](R)-PK11195 positron emission tomography imaging of activated microglia in vivo in Rasmussen's encephalitis. Neurology. 53, 2199-2203.

Doorduin, J., de Vries, E.F., Willemsen, A.T., de Groot, J.C., Dierckx, R.A., Klein, H.C., 2009. Neuroinflammation in schizophrenia-related psychosis: a PET study. Journal of nuclear medicine : official publication, Society of Nuclear Medicine. 50, 1801-1807.

Kumar, A., Chugani, H.T., Luat, A., Asano, E., Sood, S., 2008. Epilepsy surgery in a case of encephalitis: use of 11C-PK11195 positron emission tomography. Pediatr. Neurol. 38, 439-442.

Silver, J., Miller, J.H., 2004. Regeneration beyond the glial scar Nature reviews. Neuroscience 5, 146-156.

Huang, W.C., Kuo, W.C., Cherng, J.H., Hsu, S.H., Chen, P.R., Huang, S.H., et al., 2006. Chondroitinase $\mathrm{ABC}$ promotes axonal re-growth and behavior recovery in spinal cord injury. Biochem. Biophys. Res. Commun. 349, 963-968.

Schwarzacher, S.W., Vuksic, M., Haas, C.A., Burbach, G.J., Sloviter, R.S., Deller, T., 2006. Neuronal hyperactivity induces astrocytic expression of neurocan in the adult rat hippocampus. Glia. 53, 704-714. Research Support, N.I.H., Extramural Research Support, Non-U.S. Gov't.

Roitbak, T., Sykova, E., 1999. Diffusion barriers evoked in the rat cortex by reactive astrogliosis. Glia 28, 40-48.

Vorisek, I., Hajek, M., Tintera, J., Nicolay, K., Sykova, E., 2002. Water ADC, extracellular space volume, and tortuosity in the rat cortex after traumatic injury. Magnetic resonance in medicine: official journal of the Society of Magnetic Resonance in Medicine/Society of Magnetic Resonance in Medicine. 48, 994-1003. 
Davson, H., Spaziani, E., 1959. The blood-brain barrier and the extracellular space of brain. The Journal of physiology. 149, 135-143.

Sykova, E., 2004. Diffusion properties of the brain in health and disease. Neurochem. Int. 45, 453-466. Review.

Seo, G.S., Aoki, J., Moriya, H., Karakida, O., Sone, S., Hidaka, H., et al., 1996. Hyaline cartilage: in vivo and in vitro assessment with magnetization transfer imaging. Radiology 201, 525-530.

Laurent, D., Wasvary, J., Yin, J., Rudin, M., Pellas, T.C., O’Byrne, E., 2001. Quantitative and qualitative assessment of articular cartilage in the goat knee with magnetization transfer imaging. Magn. Reson. Imaging 19, 1279-1286.

Toffanin, R., Mlynarik, V., Russo, S., Szomolanyi, P., Piras, A., Vittur, F., 2001. Proteoglycan depletion and magnetic resonance parameters of articular cartilage. Arch. Biochem. Biophys. 390, 235-242.

Laurent, T.C., Fraser, J.R., 1992. Hyaluronan FASEB journal : official publication of the Federation of American Societies for Experimental Biology. 6, 2397-2404.
Kim, M., Cercignani, M., 2014. Magnetization Transfer. In: Cohen-Adad, J., WheelerKingshott, C.A.M. (Eds.), Quantitative MRI of the Spinal Cord. Academic Press, pp. $164-180$.

Dombroski, T.C.D., Peixoto-Santos, J.E., Maciel, K., Baqui, M.M.A., Velasco, T.R., Sakamoto, A.C., et al., 2020. Drebrin expression patterns in patients with refractory temporal lobe epilepsy and hippocampal sclerosis. Epilepsia.

Steve, T.A., Gargula, J., Misaghi, E., Nowacki, T.A., Schmitt, L.M., Wheatley, B.M., et al., 2020. Hippocampal subfield measurement and ILAE hippocampal sclerosis subtype classification with in vivo 4.7 tesla. MRI. Epilepsy Res. 161, 106279.

Thom, M., 2014. Review: Hippocampal sclerosis in epilepsy: a neuropathology review. Neuropathol. Appl. Neurobiol. 40, 520-543. https://doi.org/10.1111/nan.12150.

Thom, M., Liagkouras, I., Martinian, L., Liu, J., Catarino, C.B., Sisodiya, S.M., 2012.

Variability of sclerosis along the longitudinal hippocampal axis in epilepsy: a post mortem study. Epilepsy Res. 102, 45-59. 